\title{
'My training prepared me for far more than just clinical dentistry'
}

\author{
By Sophie Clarke, Foundation Dentist, London
}

to ince dental practices closed their doors to patients, most foundation dentists have either assisted their practices in triaging patients, been redeployed to NHS 111 , or have been sent to other secondary care settings. Collectively there has been an impressive willingness by foundation dentists to be redeployed and contribute to the efforts to help mitigate the devastating effects of the pandemic.

I worked with a team established in March 2020 to manage the increasing demand on the London Ambulance Service, GPs and funeral directors caused by the rising death toll in the community.

The team was assembled at short notice and was a testament to the organisation and dedication of the NHS. Comprehensive training was undertaken. The role required resilience balanced with sensitivity and empathy to support, day or night, families in shock immediately following tragic and often unexpected loss of their loved ones. Dealing with families experiencing the challenges of grief in unprecedented circumstances exposed and challenged communication skills that had only recently been tested in OSCE stations.
However, it was encouraging to find that my training had prepared me for far more than just clinical dentistry. Taking detailed histories and the safe application of PPE were familiar territory, but most importantly it was the frameworks of communication, such as the Calgary-Cambridge model, ${ }^{1}$ which proved invaluable for structuring difficult conversations with bereaved families. Attributes instilled throughout the foundation training programme such as professionalism, leadership and multi-disciplinary teamwork were widely transferable for my redeployment role, and critical to meet its demands. Although often perceived as 'soft skills', it is essential that these non-clinical competencies continue to be taught rigorously within the foundation training curriculum. We received continued support throughout the entire process; the bi-weekly mentoring from Health Education England and the supportive structure of foundation training meant we had supervisors and training programme directors readily available.

Since COVID-19 deaths in the community have dramatically reduced in London, I now

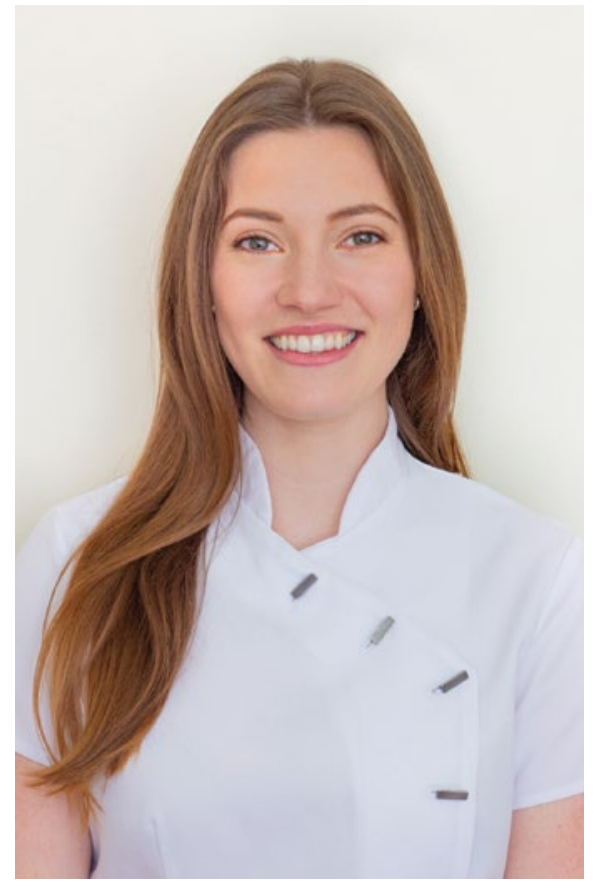

return to foundation training reflecting on the robust support and varied opportunities provided to foundation trainees. Recent graduates should be confident in their training, and no doubt their redeployment experiences, that have contributed to their development as rounded healthcare professionals; the comprehensive education in wider non-clinical skills has proved indispensable in wholly unexpected circumstances.

\section{Reference}

1. Kurtz S M, Silverman J D, Benson J, Draper J. Marrying content and process in clinical method teaching enhancing the Calgary-Cambridge guides. Acad Med 2003; 78: 802-809.

\section{Conference moved to 2021}

CloserStill Media, organiser of the British Dental Conference and Dentistry Show, have taken the decision to postpone the British Dental Conference and Dentistry Show (BDCDS). The 2020 event will now take place on Friday 21 and Saturday 22 May 2021 at the Birmingham NEC, hall 5.

Martin Woodrow, Chief Executive of the British Dental Association (BDA), said: 'It's a real shame that we can't hold what would have been an excellent conference and exhibition this year, but in the circumstances, postponing the 2020 event has to be the right thing to do and I'm sure the 2021 event will be bigger and better than ever!' In the meantime the event organisers will be focussing on how they can support the profession as they prepare to return to their practice and resume some normality during a very testing time for dentistry.

The BDCDS is the UK's leading two-day exhibition and conference for dental professionals, providing clinical, leadership and practical training via an extensive

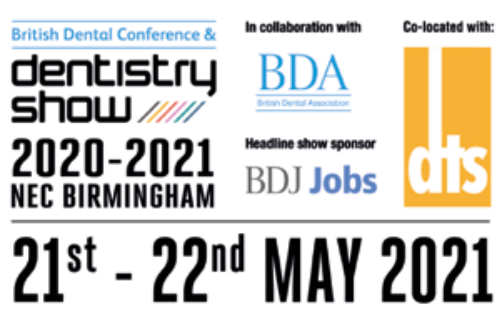

educational programme and hands-on workshops. It is the UK's largest event of its kind, attracting over 9,000 unique delegates and over 400 exhibitors.

The May 2021 conference will still be co-located with The Dental Technology Showcase. 